\title{
Stark broadening of spectral lines of multicharged ions of astrophysical interest. XXIII. Be III ${ }^{\star}$
}

\author{
M. S. Dimitrijević ${ }^{1,2}$, M. Dačici ${ }^{1,2}$, Z. Cvetković ${ }^{1,2}$, and S. Sahal-Bréchot ${ }^{3}$ \\ 1 Astronomical Observatory, Volgina 7, 11160 Belgrade, Serbia, Yugoslavia \\ 2 Institute Isaac Newton of Chile, Yugoslavia Branch \\ 3 LERMA, UMR CNRS 8112, Observatoire de Paris-Meudon, 92195 Meudon, France
}

Received 6 November 2002 / Accepted 17 December 2002

\begin{abstract}
Using a semiclassical perturbation method, we have calculated electron-, proton-, and ionized helium-impact line widths and shifts for $52 \mathrm{Be}$ III multiplets as a function of temperature and perturber density. Electron temperatures are $10000 \mathrm{~K}$; $20000 \mathrm{~K} ; 50000 \mathrm{~K} ; 100000 \mathrm{~K} ; 200000 \mathrm{~K}$ and $300000 \mathrm{~K}$ and perturber densities are from $10^{11} \mathrm{~cm}^{-3}$ up to $10^{21} \mathrm{~cm}^{-3}$. The obtained results have been used for discussion of regularities and systematic trends along spectral series.
\end{abstract}

Key words. lines: profiles - atomic data - atomic processes - line: formation

\section{Introduction}

The astrophysical importance of light element ions line profile study is obvious, since such lines are present in stellar atmospheres. Such results are of interest e.g. for numerical modeling of stellar atmospheres or abundance determinations. The Be III Stark broadening parameters are additionally interesting for problems correlated with nucleogenesis, mixing between atmospheres and the interior, stellar structure and evolution (see e.g. Boesgaard 1988), and opacity calculations (Seaton 1988). Moreover, Stark broadening parameters of Be III lines are of interest for the investigation and diagnostic of laboratory and laser-produced plasma, as well as for the consideration of regularities and systematic trends. First experimental investigations of Be III plasma spectral line shapes, have been made by Fraenkel et al. (1968) who investigated high energy satellites in Be III spectrum and Malvezi et al. (1975) who investigated high-density laser-produced Be plasma. Rosznayi (1977) performed a theoretical study of Be III lines Stark broadening for a high density plasma with $k T=21 \mathrm{eV}$, and atom density $10^{19}-10^{21} \mathrm{~cm}^{-3}$. In Dimitrijević \& Konjević (1981) and Dimitrijević (1988a,b) Stark widths of Be III lines have been calculated within the semiempirical method (Griem 1968), the modified semiempirical method (Dimitrijević \& Konjević 1980), the symplified semiclassical method (Griem 1974, Eq. (526)) and its modification (Dimitrijević \& Konjević 1980). In Dimitrijević \& Sahal-Bréchot (1996a,b) Stark

Send offprint requests to: M. S. Dimitrijević, e-mail: mdimitrijevic@aob.bg.ac.yu

* Table 1 is only available in electronic form at the CDS via anonymous ftp to cdsarc.u-strasbg.fr $(130.79 .128 .5)$ or via http://cdsweb.u-strasbg.fr/cgi-bin/qcat?/A+A/400/791 broadening parameters of 12 Be III spectral lines have been obtained within the semiclassical perturbation approach (Sahal-Bréchot 1969a, b; see also Sahal-Bréchot 1974, 1991; Fleurier et al. 1977; Dimitrijević \& Sahal-Bréchot 1984; Dimitrijević et al. 1991; Dimitrijević \& Sahal-Bréchot 1995a,b). However, the recently published analysis of the spectrum and term system of Be III (Jupén et al. 2001) enables the calculation of Stark broadening parameters for 52 additional multiplets, with the standard accuracy. The aim of this work is to determine this additional parameters in order to extend the set of available Stark broadening data.

Obtained data are also of interest for investigations of regularities and systematic trends and have been used here for the investigations of such trends along spectral series. Results of such investigations are of interest for acquisition of new data by interpolation and for critical evaluation of existing experimental and theoretical data. Moreover, the obtained results are of interest for the investigation of regularities along the helium isoelectronic sequence since besides the results for Be III obtained here and in Dimitrijević \& Sahal-Bréchot (1996a,b), the results for Li II (Dimitrijević \& Sahal-Bréchot 1996c), C V (Dimitrijević \& Sahal-Bréchot 1996d), O VII (Dimitrijević \& Sahal-Bréchot 1998a), Na X (Dimitrijević \& Sahal-Bréchot 1998b) and Si XIII (Dimitrijević \& Sahal-Bréchot 1998c), have been published also.

This paper is the twenty third of a series devoted to the calculation of Stark broadening parameters of spectral lines of multicharged ions (see Dimitrijević \& Sahal-Bréchot 1998b and references therein, Dimitrijević \& Sahal-Bréchot 1988a,d; 1999), as the continuation of our previous research. A review of obtained results is given e.g. in Dimitrijević (1996). 


\section{Results and discussion}

The semiclassical perturbation formalism used here, has been reviewed briefly e.g. in Dimitrijević \& Sahal-Bréchot (1995a, 1996c). Energy levels for Be III have been taken in Jupén et al. (2001). Oscillator strengths have been calculated by using the method of Bates \& Damgaard (1949) and the tables of Oertel $\&$ Shomo (1968). For higher levels, the method described by van Regemorter et al. (1979) has been used.

The results for 52 Be III multiplets, for Stark broadening due to electron-, proton-, and ionized helium-impacts are shown in Table 1 (accessible only in electronic form) for perturber densities $10^{11} \mathrm{~cm}^{-3}-10^{21} \mathrm{~cm}^{-3}$ and temperatures from 10000 up to $300000 \mathrm{~K}$. Stark broadening parameters for densities lower than for tabulated values, are linear with perturber density. We also specify a parameter C (Dimitrijević \& Sahal-Bréchot 1984), which gives an estimate for the maximum perturber density for which the line may be treated as isolated, when it is divided by the corresponding full width at half maximum. For each value given in Table 1 the collision volume $(V)$ multiplied by the perturber density $(N)$ is much less than one and the impact approximation is valid (Sahal-Bréchot 1969a,b). Values for $N V>0.5$ are not given and values for $0.1<N V \leq 0.5$ are denoted by an asterisk. When the impact approximation is not valid, the ion broadening contribution may be estimated by using quasistatic approach (Sahal-Bréchot 1991 or Griem 1974). In the region between where neither of these two approximations is valid, a unified type theory should be used. For example in Barnard et al. (1974), a simple analytical formula for such a case is given. The accuracy of the results obtained decreases when broadening by ion interactions becomes important.

In Figs. 1 and 2 the electron-impact full half widths and shifts for Be III $n_{\mathrm{f}} \mathrm{s}^{1} \mathrm{~S}-n_{\mathrm{i}} \mathrm{p}^{1} \mathrm{P}^{\mathrm{o}}$ lines as a function of $n_{\mathrm{i}}$ for different $n_{\mathrm{f}}$, for $T=50000 \mathrm{~K}$ at $N_{\mathrm{e}}=10^{17} \mathrm{~cm}^{-3}$ is shown. Here, $n_{\mathrm{i}}$ is the principal quantum number of initial (upper), and $n_{\mathrm{f}}$ of the final (lower) level of the considered transition. We can see gradual change of Stark broadening parameters within particular $n_{\mathrm{f}} \mathrm{s}^{1} \mathrm{~S}-n_{\mathrm{i}} \mathrm{p}^{1} \mathrm{P}^{\mathrm{o}}$ spectral series, permitting the interpolation of new data or critical evaluation of mutual consistency of existing data as in our previous analyses (see e.g. Dimitrijević \& Sahal-Bréchot 1996c). Exceptions of such behaviour have been analysed e.g. in Tankosić et al. (2001). As an addition to previous analyses (e.g. Dimitrijević \& Sahal-Bréchot 1996c), where only one Li II $2 s^{3} \mathrm{~S}-n \mathrm{p}^{3} \mathrm{P}^{\mathrm{o}}$ series have been analysed in Figs. 1-2 are considered all calculated transitions belonging to different Be III $n_{\mathrm{f}} \mathrm{s}^{1} \mathrm{~S}-n_{\mathrm{i}} \mathrm{p}^{1} \mathrm{P}^{\mathrm{o}}$ series. One can be seen that differences between corresponding members with the same initial (upper) level of different spectral series are not large and increase gradually with the increase of the principal quantum number of the final (lower) level. Consequently, rough estimation of the mutual consistency of existing data or of an approximate value for a member of a different spectral series is possible in considered case on the basis of considered regularities if the upper (initial) level of the investigated transition is the same as for members of other spectral series with known Stark broadening parameters. Such regular behaviour of Stark broadening parameters within considered spectral series is the

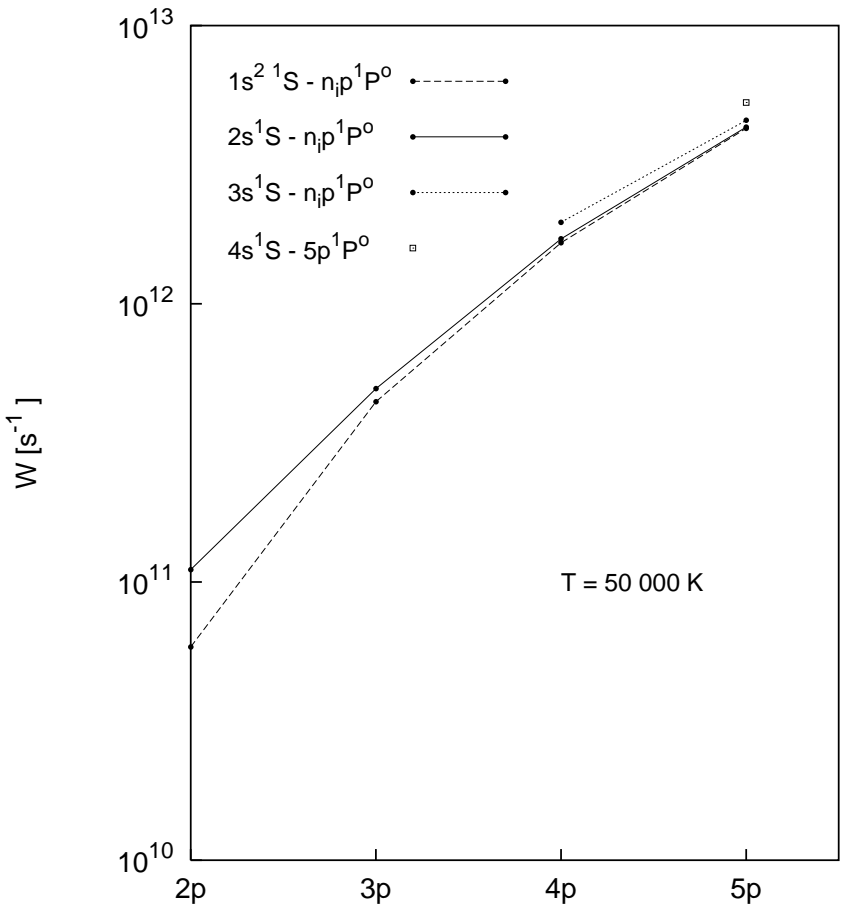

Fig. 1. Electron-impact full half widths (in angular frequency units) for Be III $n_{\mathrm{f}} \mathrm{s}^{1} \mathrm{~S}-n_{\mathrm{i}} \mathrm{p}^{1} \mathrm{P}^{\mathrm{o}}$ lines as a function of $n_{\mathrm{i}}$ for different $n_{\mathrm{f}}$, for $T=$ $50000 \mathrm{~K}$ at $N_{\mathrm{e}}=10^{17} \mathrm{~cm}^{-3}$. Here, $n_{\mathrm{i}}$ is the principal quantum number of initial (upper), and $n_{\mathrm{f}}$ of the final (lower) level of the considered transition.

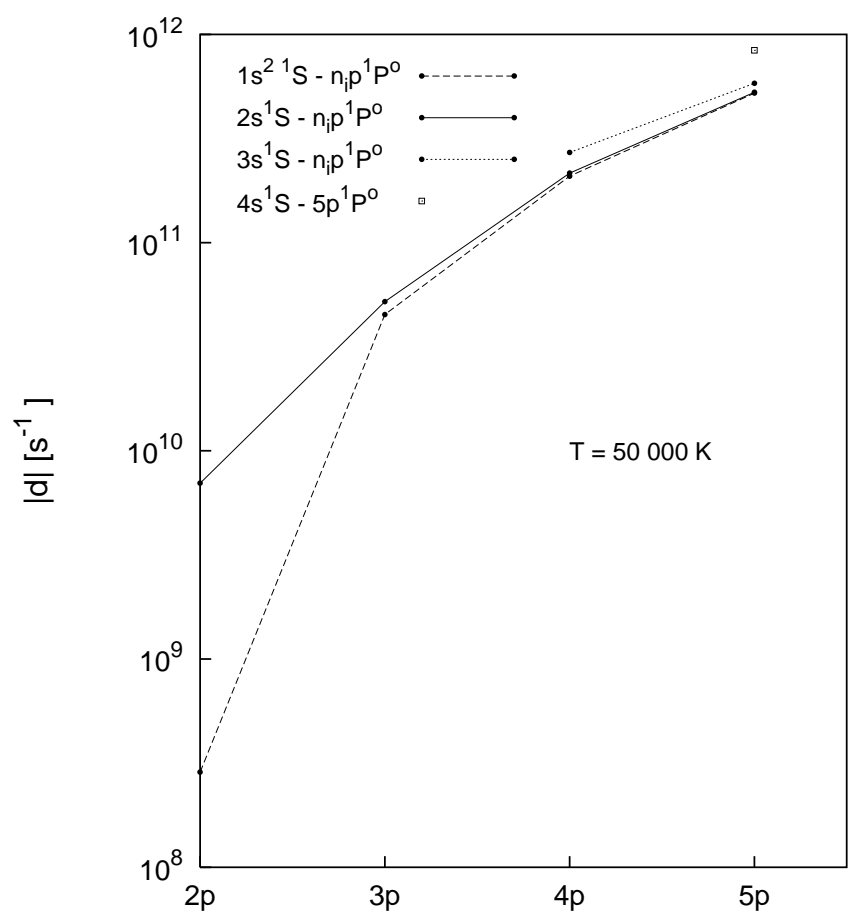

Fig. 2. As in Fig. 1 but for electron-impact shift.

consequence of the gradual change of the energy separations between the initial (upper) level and the principal perturbing levels, shown in Fig. 3. It is of interest to determine experimentally Stark broadening parameters for several members of 


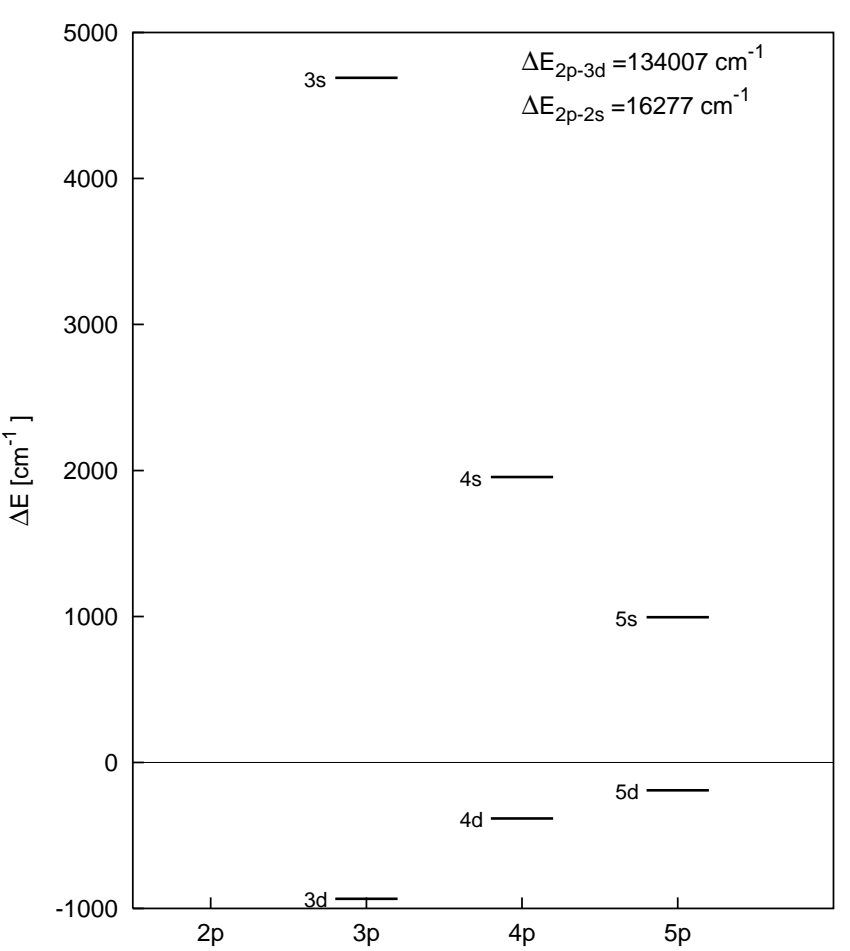

Fig. 3. Energy separations between the upper level $n_{\mathrm{i}} \mathrm{p}^{1} \mathrm{P}^{\mathrm{o}}$ and the principal perturbing levels for Be III $n_{\mathrm{f}} \mathrm{s}^{1} \mathrm{~S}-n_{\mathrm{i}} \mathrm{p}^{1} \mathrm{P}^{\mathrm{o}}$ lines. Here, $n_{\mathrm{i}}$ is the principal quantum number of initial (upper), and $n_{\mathrm{f}}$ of the final (lower) level of the considered transition.

a Be III spectral series in order to test experimentally the possibilities of established regularities within spectral series for prediction of new data and checking the reliability and mutual consistency of existing ones.

Acknowledgements. This work is a part of the project "Influence of collisional processes on astrophysical plasma lineshapes", supported by Ministry of Science, Technologies and Development of Serbia. The research was supported also by the Fonds zur Förderung der wissenschaftlichen Forschung (Project S7303-AST).

\section{References}

Barnard, A. J., Cooper, J., \& Smith, E. W. 1974, J. Quant. Spectrosc. Radiative Transfer, 14, 1025

Bates, D. R., \& Damgaard, A. 1949, Trans. Roy. Soc. London, Ser. A, 242,101
Boesgaard, A. M. 1988, Vistas Astron., 31, 167

Dimitrijević, M. S. 1996, Zh. Prikl. Spektrosk., 63, 810

Dimitrijević, M. S. 1988a, A\&AS, 76, 53

Dimitrijević, M. S. 1988b, Bull. Obs. Astron. Belgrade, 139, 31

Dimitrijević, M. S., \& Konjević, N. 1980, J. Quant. Spectrosc. Radiat. Transfer, 24, 451

Dimitrijević, M. S., \& Konjević, N. 1981, in Spectral Line Shapes, ed. B. Wende, W. de Gryuter, \& Co. Berlin, New York, 221

Dimitrijević, M. S., \& Sahal-Bréchot, S. 1984, J. Quant. Spectrosc. Radiat. Transfer, 31, 301

Dimitrijević, M. S., \& Sahal-Bréchot, S. 1995a, Phys. Scr., 52, 41

Dimitrijević, M. S., \& Sahal-Bréchot, S. 1995b, A\&AS, 109, 551

Dimitrijević, M. S., \& Sahal-Bréchot, S. 1996a, A\&AS, 119, 369

Dimitrijević, M. S., \& Sahal-Bréchot, S. 1996b, Bull. Astron. Belgrade, 153, 101

Dimitrijević, M. S., \& Sahal-Bréchot, S., 1996c, Phys. Scr., 54, 50

Dimitrijević, M. S., \& Sahal-Bréchot, S. 1996d, A\&AS, 115, 351

Dimitrijević, M. S., \& Sahal-Bréchot, S. 1998a, A\&AS, 131, 141

Dimitrijević, M. S., \& Sahal-Bréchot, S. 1998b, A\&AS, 130, 539

Dimitrijević, M. S., \& Sahal-Bréchot, S. 1998c, A\&AS, 129, 155

Dimitrijević, M. S., \& Sahal-Bréchot, S. 1998d, A\&AS, 131, 143

Dimitrijević, M. S., \& Sahal-Bréchot, S. 1999, A\&AS, 133, 227

Dimitrijević, M. S., Sahal-Bréchot, S., \& Bommier, V. 1991, A\&AS, 89,581

Fleurier, C., Sahal-Bréchot, S., \& Chapelle, J. 1977, J. Quant. Spectrosc. Radiat. Transfer, 17, 595

Fraenkel, B. S., Goldsmith, S., \& Feldman, U. 1968, Phys. Lett. A, 27, 111

Griem, H. R. 1968, Phys. Rev., 165, 258

Griem, H. R. 1974, Spectral Line Broadening by Plasmas (Academic Press, New York)

Jupén, C., Meigs, A., von Hellermann, M., et al. 2001, Phys. Scr., 64, 566

Malvezi, A. M., Janitti, E., \& Tondello, G. 1975, Opt. Commun., 13, 307

Oertel, G. K., \& Shomo, L. P. 1968, ApJS, 16, 175

Rosznayi, B. F. 1977, J. Quant. Spectrosc. Radiat. Transfer, 17, 77

Sahal-Bréchot, S. 1969a, A\&A, 1, 91

Sahal-Bréchot, S. 1969b, A\&A, 2, 322

Sahal-Bréchot, S. 1974, A\&A, 35, 321

Sahal-Bréchot, S. 1991, A\&A, 245, 322

Seaton, M. J. 1988, J. Phys. B, 21, 3033

Tankosić, D., Popović, L. Č., \& Dimitrijević, M. S. 2001, Phys. Scr., 63,54

van Regemorter, H., Hoang Binh Dy, \& Prud'homme, M. 1979, J. Phys. B, 12, 1073 